\title{
Perspectivas sobre Crecimiento Económico y Medio Ambiente: Curva Ambiental de Kuznets en la Alianza del Pacífico
}

\author{
Perspectives on Economic Growth and the \\ Environment: Environmental Curve of Kuznets in \\ the Pacific Alliance
}

\author{
Vega-Quezada, Cristhian Antonio \\ Universidad Técnica de Machala \\ (Machala - Ecuador) \\ cvega@utmachala.edu.ec \\ Varela-Veliz, Gregory Darío \\ Universidad Técnica de Machala \\ (Machala - Ecuador) \\ gredavave@gmail.com
}

Martínez-Jiménez, Diego Armando Universidad Técnica de Machala

(Machala - Ecuador) damartinez_est@utmachala.edu.ec

Soto-González, Carlos Omar Universidad Técnica de Machala (Machala - Ecuador) csoto@utmachala.edu.ec

Revista Cumbres Vol.5 №2

Versión impresa ISSN 1390-9541

Versión electrónica ISSN 1390-3365

http://investigacion.utmachala.edu.ec/revistas/index.php/Cumbres 


\title{
RESUMEN
}

Este artículo tiene el objetivo principal de demostrar la existencia o no, de la hipótesis de la curva ambiental de Kuznets (CAK) en forma de U invertida en los países de la Alianza del Pacífico. Se consideraron los cuatro países que conforman el bloque comercial como ámbito de estudio, aplicando herramientas metodológicas como, los modelos Autorregresivos de Rezagos Distribuidos (ARDL). Se estimaron las relaciones existentes entre el crecimiento económico, como variable independiente, y las emisiones de $\mathrm{CO} 2$, como variable dependiente, en el corto y largo plazo. El periodo de análisis comprendió una serie de tiempo desde el año 1991 hasta el 2017. Se consideraron cinco variantes de los modelos propuestos incluyendo variables como: la población, la inversión extranjera directa (IED), el desempleo, el coeficiente de Gini y las exportaciones de bienes y servicios. El principal aporte de esta investigación radica en presentar la relación existente entre las principales variables de crecimiento económico y las emisiones de $\mathrm{CO} 2$ en los miembros de la alianza económica, en donde, México evidenció un mayor cumplimiento de la hipótesis de la curva ambiental de Kuznets de acuerdo a los datos analizados en el periodo de estudio.

Palabras clave: Curva ambiental de Kuznets, crecimiento económico, emisiones de CO2 per cápita, ARDL

\begin{abstract}
This article has the main objective of demonstrating the existence or not of the hypothesis of the environmental Kuznets Curve (EKC) in the form of an inverted $U$ in the countries of the Pacific Alliance. The four countries that make up the commercial block were considered as a field of study, applying methodological tools such as, the Autoregressive Distributed Lag (ARDL) models. The relationships between economic growth, as an independent variable, and $\mathrm{CO} 2$ emissions, as a dependent variable, were estimated in the short and long term. The period of analysis included a time-series from 1991 to 2017. Five variants of the proposed models were considered, including variables such as: population, foreign direct investment (FDI), unemployment, Gini coefficient and exports of goods and services. The main contribution of this research lies in presenting the relationship between the main variables of economic growth and $\mathrm{CO} 2$ emissions in the members of the economic alliance, where Mexico showed greater compliance with the environmental Kuznets curve hypothesis according to the data analyzed in the study period.
\end{abstract}

Keywords: Environmental Kuznets curve, economic growth, CO2 emissions per capita, ARDL

\section{Cumbres}




\section{INTRODUCCIÓN}

En los últimos años, el tema de la conservación ambiental ha sido plenamente abordado por la población en general, provocando debate en torno, a si las empresas a nivel mundial deben dejar de producir o mejorar sus procesos de producción, esta fricción de pensamientos ha generado que se acuerden diferentes tratados en fomento del medio ambiente, pero en muchos casos no han dado el efecto esperado.

Expertos autores de literatura económica, entre ellos, Panayotou (1993; 1997), menciona que los países que se encuentran en la transición económica, del sector primario (materia prima) al sector secundario, industrial, tal como China, Corea del Sur, Tailandia o México, están más contaminados que hace 20 o 30 años atrás. Mientras que, países considerados industriales, como Estados Unidos, Reino Unido y Japón registran una menor contaminación ambiental de la que registraban cuando optimizaron su desarrollo industrial y alcanzaron su punto de inflexión hace 20 o 30 años en el pasado.

El estudio realizado por Simon Kuznets (1955), donde compara el crecimiento económico, y la inequidad de los ingresos en (1991) permitió a los autores Grossman and Krueger dar origen a la hipótesis de la Curva Ambiental de Kuznets, de ahora en adelante (CAK). De acuerdo a la revisión literaria de diferentes investigadores Stern (2004); Lau, Choong, and Eng (2014) ; Baek (2015); Apergis \& Ozturk (2015) ; Al-Mulali, Solarin, and Ozturk (2016), la CAK relaciona los indicadores de degradación ambiental, y el PIB per cápita, mencionando que en el corto plazo, los países emergentes en miras de convertirse en economías de primer mundo, incrementan sus indicadores de degradación ambiental, hasta llegar a un punto de inflexión, donde el incremento de su ingreso per cápita induce a una reducción de los niveles de inequidad en sus economías, y por ende la disminución de los indicadores de contaminación medioambiental, tomando así forma de una U invertida que demuestra lo antes mencionado.

La presente investigación está dividida en tres partes fundamentales, en la primera se realiza una breve introducción sobre la actualidad ambiental en el planeta y la hipótesis de la curva ambiental de Kuznets (CKA). En la siguiente parte se analizan las variables propuestas con el modelo ARDL, donde se presentan la evidencia de la curva ambiental en los países de la Alianza del Pacífico. Finalmente se presentan las conclusiones de la investigación de acuerdo a los resultados presentados.

\section{Emisiones de dióxido de carbono (co2) a nivel mundial}

El Dióxido de Carbono (CO2) es un gas no tóxico compuesto por dos moléculas de oxígeno y una de carbono. Según datos del The World Bank (2018), este gas es el de mayor abundancia en el planeta, con una representación numérica de 33 848,39 millones de Tm. Es importante considerar que el año 2014, solo 3 países emiten más de la mitad de este gas. Estos son China, Estados Unidos y la India, juntos estos países emanaron 17 784,58 millones de Tm de CO2, representando el 52,54\% a nivel mundial. 
Debido a su alto desarrollo industrial, en el año 2014, se registra que China y Estados Unidos, son los países que mas $\mathrm{CO} 2$ emiten al medio ambiente con 10 291,93 millones de Tm y 5254,28 millones de Tm, respectivamente, figurando cifras de 30,41\% y 15,52\%. En un alejado tercer lugar, le sigue la India con 2238,38 millones de Tm de CO2, representando 6,61\% de emisiones. A nivel de la región de América Latina, el principal emisor es Brasil con 529,81 millones de Tm del gas contaminante.

\section{Emisiones de dióxido de carbono (co2) en la alianza del pacífico}

Según cifras del The World Bank (2018) , en el año 2014, el bloque comercial registro emisiones de $\mathrm{CO} 2$ por 708,67 millones de Tm, que representan el 2,09\% de gas contaminante a nivel mundial, a continuación en la tabla 1 se detalla el orden de los países.

Tabla 1. Emisiones de CO2 en la Alianza del Pacífico (millones de Tm y Tm per cápita, de mayor a menor).

\begin{tabular}{|l|l|l|l|l|l|}
\hline $\mathrm{N}^{\circ}$ & Países & $\begin{array}{l}\text { Emisiones de } \\
\text { CO2 (Millones } \\
\text { de Tm) }\end{array}$ & $\%$ & $\begin{array}{l}\text { Emisiones de } \\
\text { CO2 (Tm per } \\
\text { cápita) }\end{array}$ & $\mathrm{N}^{\circ}$ \\
\hline 1 & México & 480,27 & 1,42 & 3,86 & 2 \\
\hline 2 & Colombia & 84,09 & 0,25 & 1,75 & 4 \\
\hline 3 & Chile & 82,56 & 0,24 & 4,68 & 1 \\
\hline 4 & Perú & 61,74 & 0,18 & 1,99 & 3 \\
\hline
\end{tabular}

Fuente: The World Bank (2018)

Como podemos notar en la tabla anterior, el país que más emitió fue México con 480,27 millones de Tm de CO2 representando el 1,42\% del total mundial, mientras que Perú fue el país menos contaminado de los 4 miembros, registrando 61,74 millones de Tm de CO2 figurando con un 0,18\% a nivel mundial. A su vez, notamos que las emisiones de CO2 fueron lideradas por Chile con 4,68 Tm por habitante, mientras que Colombia fue el que menos emisiones emana por persona con 1,75 Tm de CO2.

\section{Mecanismos de mitigación ambiental: mecanismos de desarrollo limpio (mdl) en la Alianza del Pacífico}

Los mecanismos de desarrollo por el cual los países industrializados utilizan para invertir en proyectos de reducción de emisiones de Gases de efecto invernadero (GEI) en países en desarrollo o en vía de desarrollo, en donde los países industrializados como contrapartida reciben un pago o transferencia de Certificado de emisiones reducidas (CER's), basados en el utilidad del proyecto (Méndez \& Perugache, 2012), cuyo CER's pueden ser utilizados como respaldo del cumplimiento de sus compromiso para la reducción de las emisiones de los GEI (Sanabria-Aguirre \& Hurtado-Aguirre, 2013).

Los mecanismos de desarrollo limpio forman parte de un grupo de mecanismos establecidos dentro del Protocolo de Kyoto (1998), estos mecanismos de flexibilidad sirven para la reducción de las emisiones de los GEI, los cuáles 
a largo plazo son fundamentales para ayudar a mitigar los efectos de la degradación ambiental (Zilio, 2012).

A continuación, en la tabla 2, se visualizan los proyectos de MDL que se encuentran registrados por los miembros de la Alianza del Pacífico.

Tabla 2. Total de Proyectos de MDL en la Alianza del Pacífico (orden alfabético).

\begin{tabular}{|l|l|l|}
\hline Países & Total de Proyectos de MDL & Reducción de Emisiones (Tm/año) \\
\hline Chile & 110 & $12^{\prime} 843904$ \\
\hline Colombia & 68 & $8^{\prime} 191100$ \\
\hline México & 204 & $21^{\prime} 191735$ \\
\hline Perú & 64 & $10^{\prime} 983039$ \\
\hline
\end{tabular}

Fuente: (UNFCCC, 2017)

En la anterior tabla, se denota que en la Alianza del Pacífico se encuentran registrados 446 proyectos de MDL que juntos en total ayudan a mitigar las emisiones de $\mathrm{CO} 2$ en 53'21 millones deTm al año, a la vanguardia del bloque se encuentra México con 204 proyectos que dejarán de emitir 21,84 millones de Tm al año, mientras que, el país que menos proyectos registra es Perú con 64 proyectos que le ayudan a mitigar las emisiones de $\mathrm{CO} 2$ a la atmósfera en 10,98 millones de Tm por año. Es considerable destacar que los países de grupo comercial están trabajando en cuento a la protección del medio ambiente.

\section{MATERIALES Y MÉTODOS}

El objetivo primordial de esta investigación, es poder demostrar la evidencia de la hipótesis empírica de la curva ambiental de Kuznets (CAK) en los países que forman parte de la Alianza del Pacífico, mediante la aplicación de modelos ARDL (Autorregresivo de Rezagos Distribuidos), en donde se analiza a las emisiones de CO2 per cápita como variable dependiente y como variable independiente, el ingreso per cápita (PIB per cápita). Además se consideran variables explicativas adicionales como, el coeficiente Gini, la población, la inversión extranjera directa (IED), el desempleo y la exportación de bienes y servicios (EBS), comprendidos en un período de 1991 al 2017, cuyo datos fueron obtenidos de la base de datos en línea de los indicadores de desarrollo del Banco Mundial (2017), aplicando el modelo ARDL mediante el software estadístico STATA 14. Se estiman cinco modelos diferentes, detallados a continuación.

En el modelo uno (M1), la variable dependiente fue las emisiones de CO2 per cápita, la variable independiente el PIB per cápita y la variable explicativa el coeficiente Gini. En el modelo dos (M2), la variable dependiente fue las emisiones de CO2 per cápita, la variable independiente el PIB per cápita y la variable explicativa la población. El modelo tres (M3), se consideró, las emisiones de $\mathrm{CO} 2$ per cápita como variable dependiente, la variable independiente el PIB per cápita y la variable explicativa la IED. En el modelo cuatro (M4), las emisiones de CO2 per cápita es la variable dependiente, la variable 
independiente el PIB per cápita y la variable explicativa el Desempleo. Y finalmente, se propone el modelo cinco (M5), siendo la variable dependiente las emisiones de CO2 per cápita, la variable independiente es el PIB per cápita, y la variable explicativa las exportaciones de bienes y servicios (EBS).

\section{RESULTADOS}

\section{Modelo para el crecimiento económico y las emisiones de dióxido de car- bono (Caso Alianza del Pacífico).}

Como primer punto, se determinó las pruebas de cambio estructural, a través del test de Suma acumulativa de multivariables (Cusum por sus siglas en inglés) para verificar la existencia o no de cambios de estructura económica que puedan afectar al modelo en base al tiempo analizado (Diaz del Rio, 2015; Moreno, 2015), test aplicado para todos los países que conforman la Alianza del Pacífico, así como se puede apreciar en la figura 1.

Fig. 1. Test de cusum para los cuatro países de la Alianza del Pacífico.

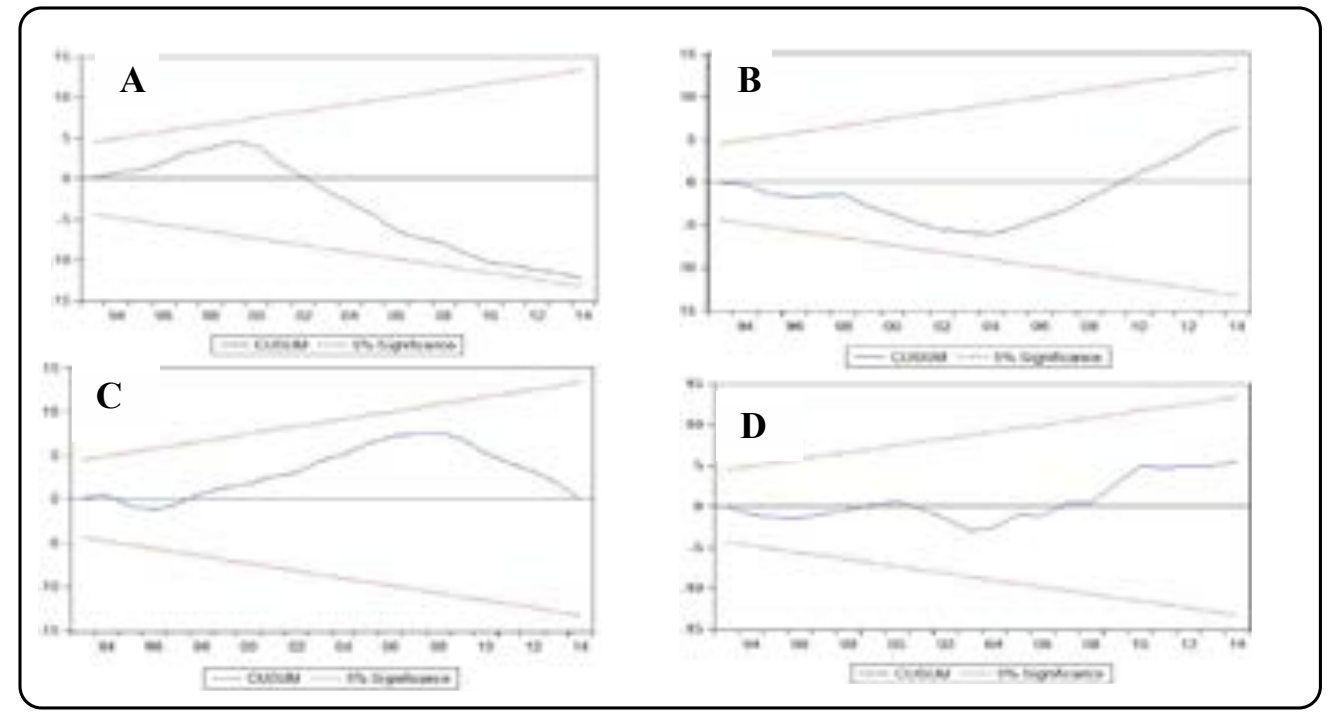

Nota: Chile (A), Colombia (B), México (C), Perú (D).

Se puede determinar que en el test de Cusum para los cuatro países de la Alianza del Pacífico, no se observa que exista un cambio estructural, ya que todas las sumas acumulativas de estos cuatro países, se encuentra dentro de los limites esperados, demostrando una situación de estabilidad económica en los modelos.

En la siguiente tabla 3, se expresan las estimaciones de corto y largo plazo de los modelos ARDL para estos países. Se puede observar que el M2, M3 y M5 son los modelos más idóneos para determinar la existencia de la hipótesis de la CAK, teniendo una relación positiva a corto plazo y una rela- 
ción negativa a largo plazo, demostrando así el cumplimiento de la evidencia empírica de U invertida entre las emisiones de CO2 per cápita y el PIB per cápita en los países de Chile, Colombia, México y Perú:

- En donde en Chile, en el corto plazo, si el PIB per cápita aumenta el 1\%, las emisiones de CO2 per cápita aumentaran en 0,75\%. Mientras que, si la exportación de bienes y servicios aumenta en una unidad porcentual, las emisiones de CO2 per cápita ascienden en 1,30\%. Por otro lado, las estimaciones de largo plazo demuestran que, si el PIB per cápita sube 1\%, las emisiones de CO2 per cápita disminuyen el 0,07\%, cumpliéndose así la hipótesis de la existencia de la EKC. Y a su vez que, si las exportaciones de bienes y servicios crecen, las emisiones de CO2 per cápita disminuyen en $0,60 \%$ respectivamente.

- Por otro lado, en Colombia, en el corto plazo, si el PIB per cápita aumenta el 1\%, las emisiones de CO2 per cápita aumentaran en 1,69\%. Mientras que, si la inversión extranjera directa aumenta, las emisiones de CO2 per cápita ascienden en $0,12 \%$. Por otro lado, las estimaciones de largo plazo demuestran que, si el PIB per cápita sube 1\%, las emisiones de CO2 per cápita disminuyen el 0,010\%, cumpliéndose así la hipótesis de la existencia de la EKC. Y finalmente, si la inversión extranjera directa aumenta, las emisiones de CO2 per cápita disminuyen en 0,11\%.

- Mientras que, en México en el corto plazo, si el PIB per cápita aumenta el $1 \%$, las emisiones de CO2 per cápita aumentaran en 0,51\%. Por otro lado, las estimaciones de largo plazo demuestran que, si el PIB per cápita sube $1 \%$, las emisiones de CO2 per cápita disminuyen el 1,06\%, cumpliéndose así la hipótesis de la existencia de la EKC. Finalmente, si la población crece, las emisiones de CO2 per cápita disminuyen en 1,24 veces.

- Y finalmente en Perú, en el corto plazo, si el PIB per cápita aumenta el 1\%, las emisiones de CO2 per cápita aumentaran en 3,06\%. Mientras que, si la población aumenta, las emisiones de CO2 per cápita aumenta en 1,89 veces. Por otro lado, las estimaciones de largo plazo demuestran que, si la población aumenta, las emisiones de CO2 per cápita disminuyen el 0,77 veces, cumpliéndose así la hipótesis de la existencia de la CAK.

Tabla 3. Estimaciones de Corto y Largo plazo en los países miembros de la Alianza del Pacífico.

\begin{tabular}{|c|c|c|c|c|}
\hline VARIABLES & M5 (Chile) & M3 (Colombia) & M2 (México) & M2 (Perú) \\
\hline \multicolumn{5}{|l|}{$\mathrm{ADF}$} \\
\hline dlnCO2perCapita & $\begin{array}{l}-2,0993 * \\
(0,4036)\end{array}$ & $\begin{array}{l}-1,6616^{*} \\
(0,2601)\end{array}$ & $\begin{array}{l}-2,2722 * \\
(0,4559)\end{array}$ & $\begin{array}{l}-3,6605^{*} \\
(0,5857)\end{array}$ \\
\hline \multicolumn{5}{|l|}{ LR } \\
\hline InPIBperCapita & $\begin{array}{l}-0,0741 * \\
(0,1072)\end{array}$ & $\begin{array}{l}-0,0069 * \\
(0,0016)\end{array}$ & $\begin{array}{l}-1,0641^{*} \\
(0,0098)\end{array}$ & $\begin{array}{l}1,0467 * 3 \\
(1,0899)\end{array}$ \\
\hline dlnPOBLACION & - & - & $\begin{array}{l}-1,2924 * * * \\
(0,5430)\end{array}$ & $\begin{array}{l}-0,7744^{*} \\
(0,2628)\end{array}$ \\
\hline dlnIED & - & $\begin{array}{l}-0,1057 * * * \\
(0,0530)\end{array}$ & - & - \\
\hline
\end{tabular}

Nota: Errores estándar en paréntesis. *Significativo al 1\%, ** Significativo al 5\%, ***Significativo al 10\% y ****Significativo al 15\%. L: rezago; D1: la diferencia; LD: rezago de la diferencia, L2D: segundo rezago de la diferencia y L3D: tercer rezago de la diferencia 


\begin{tabular}{|c|c|c|c|c|}
\hline dlnEBS & $\begin{array}{l}-0,6043^{*} \\
(0,1256)\end{array}$ & - & - & - \\
\hline \multicolumn{5}{|l|}{ SR } \\
\hline \multicolumn{5}{|c|}{ dlnCO2perCapita } \\
\hline LD. & $\begin{array}{l}0,8247 * \\
(0,2871)\end{array}$ & - & $\begin{array}{l}1,0700 * * * \\
(0,4417)\end{array}$ & $\begin{array}{l}1,8010^{*} \\
(0,4502)\end{array}$ \\
\hline \multicolumn{5}{|c|}{ InPIBperCapita } \\
\hline D1. & - & - & $\begin{array}{l}0,5081 \\
(0,3644)\end{array}$ & - \\
\hline LD. & $\begin{array}{l}0,7546 * \\
(0,2387)\end{array}$ & - & - & $\begin{array}{l}3,0940 * \\
(0,7098)\end{array}$ \\
\hline L3D. & - & $\begin{array}{c}1,6933 * \\
(0,5864)\end{array}$ & - & - \\
\hline \multicolumn{5}{|c|}{ dlnPOBLACION } \\
\hline D1. & - & - & - & $\begin{array}{c}1,8856 \\
(1,6260) \\
\end{array}$ \\
\hline \multirow[t]{2}{*}{ LD. } & - & - & $\begin{array}{c}4,1410 * * \\
(1,1480)\end{array}$ & \\
\hline & - & & & \\
\hline \multicolumn{5}{|l|}{ dlnIED } \\
\hline L2D. & - & $\begin{array}{c}0,1234^{*} \\
(0,0486)\end{array}$ & - & - \\
\hline \multicolumn{5}{|l|}{ dlnEBS } \\
\hline D1. & $\begin{array}{c}1,3029 * \\
(0,3354)\end{array}$ & - & - & - \\
\hline _const & $\begin{array}{l}1,4203^{*} \\
(0,4052)\end{array}$ & - & $\begin{array}{l}1,4062 * \\
(0,2886)\end{array}$ & \\
\hline
\end{tabular}

Así mismo en la tabla 4, se aprecia el cumplimiento de la CAK en los países de la Alianza del Pacífico, en donde México es el país que cumple con mayor cuantía la hipótesis de la CAK, evidenciándolo en cuatro de los cinco modelos propuestos. En cambio, en la tabla 3, se observa que Chile, Colombia y Perú solo cumplen la CAK en dos modelos.

Tabla 4. Cumplimiento de la CAK en la Alianza del Pacífico.

\begin{tabular}{|l|l|l|l|l|}
\hline PAISES / VARIABLES & CHILE & COLOMBIA & MÉXICO & PERÚ \\
\hline GINI & -- & -- & -- & -- \\
\hline POBLACION & -- & -- & $X$ & $X$ \\
\hline IED & $\mathrm{X}$ & $\mathrm{X}$ & $\mathrm{X}$ & $\mathrm{X}$ \\
\hline DESEMPLEO & -- & $\mathrm{X}$ & $\mathrm{X}$ & -- \\
\hline EBS & $\mathrm{X}$ & -- & $\mathrm{X}$ & -- \\
\hline
\end{tabular}




\section{CONCLUSIONES}

Una vez analizados los datos, se concluye que en los países de la Alianza de Pacífico, existe la evidencia de la hipótesis de la curva ambiental de Kuznets (CKA), demostrando que a largo plazo sus emisiones de $\mathrm{CO} 2$ son propensas a disminuir por cada unidad porcentual que aumente su PIB per cápita.

Además, se menciona que es indispensable que los países del grupo comercial continúen proponiendo y ejecutando proyectos de MDL, ya que estos son mecanismos de mitigación ambiental y pueden ayudar a mejorar la calidad de vida de las personas, a través de un ambiente menos contaminado y también como fuentes de empleo directo e indirectos.

Es de suma importancia, generar espacios de enseñanza integral e investigación en temática ambiental, ya que los individuos somos actores fundamentales en la conservación del medio ambiente, ya que tenemos la capacidad de desarrollar una cultura de comportamiento en miras de preservar el medio ambiente.

En este trabajo se plasmó el supuesto de la CAK, pero esto no garantiza su cumplimiento, en cuanto a mitigación de las emisiones de $\mathrm{CO} 2$, si en los países de la Alianza del Pacífico no se exige rigidez en el cumplimiento de las leyes, en cuanto a la temática de protección ambiental.

\section{REFERENCIAS BIBLIOGRÁFICAS}

Al-Mulali, U., Solarin, S. A., \& Ozturk, I. (2016). Investigating the presence of the environmental Kuznets curve (EKC) hypothesis in Kenya: an autoregressive distributed lag (ARDL) approach. Natural Hazards (Vol. 80). Springer Netherlands. http://doi.org/10.1007/s11069-015-2050-x

Apergis, N., \& Ozturk, I. (2015). Testing environmental Kuznets curve hypothesis in Asian countries. Ecological Indicators, 52, 16-22. http://doi. org/10.1016/j.ecolind.2014.11.026

Baek, J. (2015). Environmental Kuznets curve for CO2 emissions: The case of Arctic countries. Energy Economics, 50, 13-17. http://doi.org/10.1016/j. eneco.2015.04.010

Diaz del Rio, I. (2015). El papel de la confianza en la evolución de la economía : evidencia empírica para el caso español El papel de la confianza en la evolución de la economía : evidencia empírica para el caso español. Universidadde Da Coruña.

Grossman, G. M., \& Krueger, A. B. (1991). Environmental Impacts of a North American Free Trade Agreement. National Bureau of Economic Research Working Paper Series, (3914), 1-57. http://doi.org/10.3386/w3914

Kuznets, S. (1955). Economic Growth and Income Inequality. The American Economic, 45(1), 1-28. Retrieved from http://www.jstor.org/ stable/1811581? Search=yes \&resultItemClick=true\&searchTex$\mathrm{t}=$ Economic \&searchText $=$ Growth\&searchText $=\mathrm{and} \&$ searchText $=\mathrm{In}-$ come\&searchText=Inequality\&searchUri=/action/doBasicSearch?Que$\mathrm{ry}=$ Economic+Growth+and+Income+Inequality\&amp;prq=\%28vo\%3A-8 
Lau, L. S., Choong, C. K., \& Eng, Y. K. (2014). Investigation of the environmental Kuznets curve for carbon emissions in Malaysia: DO foreign direct investment and trade matter? Energy Policy, 68, 490-497. http://doi. org/10.1016/j.enpol.2014.01.002

Méndez, J., \& Perugache, C. (2012). Causalidad y sensibilidad entre precios de los derechos de emisión europeos y los certificados de reducción de emisiones de mecanismos de desarrollo limpio en el mercado europeo de transacción de emisiones. Estudios Gerenciales, 28(124), 141-167. http://doi. org/10.1016/S0123-5923(12)70220-6

Moreno, I. (2015). Técnicas Avanzadas de Protección en Redes Eléctricas Inteligentes. Unidersidad De Cordoba.

Naciones Unidas. (1998). Protocolo de Kyoto de la Convención Marco de las Naciones Unidas sobre el Cambio Climático. Protocolo de Kyoto, 61702, 20. Retrieved from http://unfccc.int/resource/docs/convkp/kpspan.pdf

Panayotou, T. (1993). Empirical tests and policy analysis of environmental degradation at different stages of economic development. ILO Working Papers. Retrieved from http://ideas.repec.org/p/ilo/ilowps/292778.html

Panayotou, T. (1997). Demystifying the environmental Kuznets curve: turning a black box into a policy tool. Environment and Development Economics, 2(4), 465-484. http://doi.org/10.1017/S1355770X97000259

Sanabria-Aguirre, S. E., \& Hurtado-Aguirre, E. (2013). Emprendimiento verde en Colombia: el caso del mecanismo de desarrollo limpio (MDL). Entramado, 9(1), 38-65. Retrieved from http://www.redalyc.org/articulo. oa?id=265428385004

Stern, D. I. (2004). The Rise and Fall of the Environmental Kuznets Curve. World Development, 32(8), 1419-1439. http://doi.org/10.1016/j.worlddev.2004.03.004

The World Bank. (2018). Indicadores del desarrollo mundial. Retrieved February 19, 2018, from http://databank.bancomundial.org/data/source/ world-development-indicators

UNFCCC. (2017). Clean Development Mechanism (CDM). Retrieved from http://cdm.unfccc.int/Projects/projsearch.html

Zilio, M. (2012). Curva de Kuznets ambiental: la validez de sus fundamentos en países en desarrollo. Cuadernos de Economía, 35(99), 158-171. http://doi. org/10.1016/S0210-0266(12)70032-8 